Article

\title{
Lower Limb Inter-Joint Coordination of Unilateral Transfemoral Amputees: Implications for Adaptation Control
}

\author{
Zhi Xu ${ }^{1,2,3}$, Duo Wai-Chi Wong ${ }^{2,4} \oplus^{\circ}$, Fei Yan ${ }^{2}$, Tony Lin-Wei Chen ${ }^{2,4}$, Ming Zhang ${ }^{2,4, *}$, \\ Wen-Tao Jiang ${ }^{1,3, *}$ and Yu-Bo Fan ${ }^{5}$ \\ 1 Laboratory of Biomechanical Engineering, Department of Applied Mechanics, Sichuan University, \\ Chengdu 611065, China; hughes.xu@connect.polyu.hk \\ 2 Department of Biomedical Engineering, Faculty of Engineering, The Hong Kong Polytechnic University, \\ Hong Kong 999077, China; duo.wong@polyu.edu.hk (D.W.-C.W.); bme-fei.yan@polyu.edu.hk (F.Y.); \\ tony.l.chen@connect.polyu.hk (T.L.-W.C.) \\ 3 Institute for Disaster Management and Reconstruction, Sichuan University-The Hong Kong Polytechnic \\ University, Chengdu 610065, China \\ 4 Shenzhen Research Institute, The Hong Kong Polytechnic University, Shenzhen 518057, China \\ 5 Beijing Key Laboratory of Rehabilitation Technical Aids for Old-Age Disability, National Research Center for \\ Rehabilitation Technical Aids, Beijing 100176, China; yubofan@buaa.edu.cn \\ * Correspondence: ming.zhang@polyu.edu.hk (M.Z.); scubme@aliyun.com (W.-T.J.); \\ Tel.: +852-2766-4939 (M.Z.); +86-028-8203-7876 (W.-T.J.)
}

Received: 22 May 2020; Accepted: 10 June 2020; Published: 12 June 2020

\begin{abstract}
The gait of transfemoral amputees can be made smoother by adjusting the inter-joint coordination of both lower limbs. In this study, we compared the inter-joint coordination of the amputated and non-amputated limbs of unilateral amputees to able-bodied controls. Eight amputees and eight able-bodied control participants were recruited. Walking speed, stance-swing time ratio, joint angle, joint angular velocity, and inter-joint coordination parameters-including continuous relative phase (CRP) and decomposition index (DI)—of the lower-limb joint pairs in stance and swing phases were investigated. Similarity of the CRP between groups was evaluated using cross-correlation measures and root-mean-square, and the variability of the CRP was examined by deviation phase (DP). There were significant differences between the amputated limbs and controls in CRP of hip-knee and knee-ankle in stance and swing, DP of knee-ankle and hip-ankle in stance, and DI of hip-knee in swing. For the non-amputated limbs, there were significant differences in CRP and DP of knee-ankle, and DI of hip-knee in swing compared to controls. The amputees utilized unique inter-joint coordination patterns for both limbs-particularly the hip joint-to compensate for the support-capability impairment due to limb salvage and ensure foot placement accuracy.
\end{abstract}

Keywords: amputee gait; continuous relative phase; decomposition index; compensation strategy; phase portrait; inter-joint coordination

\section{Introduction}

The prosthetic gait is characterized by a relatively low walking speed [1], high asymmetry [2], and high metabolic cost [3]. Kinematically, concentric contraction of the hip flexors is needed to compensate for the loss of ankle plantar flexion for sufficient foot clearance [4]. During the single support phase, the ankle joint on the non-amputated side excessively plantarflexes to assist toe clearance [5]. Changes also occur in the hip, knee, and ankle joints for both the amputated and 
non-amputated limbs. These were described as a compensatory mechanism, which is an explanation for the adaptation control strategy for amputees $[5,6]$.

Motion capture systems (MOCAP) accompanied with force platforms are commonly used to investigate the locomotion of transfemoral amputees and compensatory motion. Reduced stance time and impulse was found in the prosthetic limb during level walking [2], whilst transfemoral amputees demonstrated greater prosthetic knee joint extension and moment during stair ascent $[7,8]$. There was no significant difference in intact limb loading during slope descend compared to the able-bodied control, despite the fact that transfemoral amputees walked slower and with smaller steps [9]. Carse et al. [10] aimed to identify the compensatory gait in 60 amputees and found that they lacked prosthetic knee flexion and intact side hip extension in early stance and late stance respectively, whereas propulsive force on the prosthetic limb was insufficient in late stance. Besides, evaluations of prosthetic designs were also conducted using MOCAP. For example, customized prosthetic socket [11], microprocessor-controlled prosthetic knee joints [6], and bionic prosthetic foot [12], etc.

While researchers have attempted to quantify the deviations in the prosthetic gait using kinematic or kinetic parameters [13], they overlooked the effects of the coupling and interactions between joints. Human locomotion is characterized by multiple-degrees of freedom movement, which requires different body segments and joints to cooperate with each other for achieving a precise foot trajectory [14-16]. For transfemoral amputees, the objective is to reduce the redundancy in the degrees of freedom for movement to achieve a stable, smooth, and accurate gait.

In studies on inter-joint coordination, the interactions or coupling effects were considered by evaluating the multiple degrees of freedom in the movement. These included the interactions between two joints and contemplated the timing and sequencing of the motor control. In other studies on inter-joint coordination, the effects of obstacle-crossing [17], age [18], walking speed [19], muscle fatigue [20], treadmill walking [21], and pathologies such as ankle sprain [22] and knee osteoarthritis [23]. Relevant understanding of the inter-joint coordination for transfemoral amputees could help design linkages that synergize prosthetic joints, feet, or other components, and facilitate safe and energy-saving gait for them.

Parameters commonly used to evaluate inter-joint coordination include the continuous relative phase (CRP) [24] and the decomposition index (DI) $[25,26]$. The CRP is a continuous measurement of joint interaction derived from the differences in the phase angle between two joints, while the phase angle depends on both the joint angle and the angular velocity during walking $[23,27,28]$. In decomposition movement, one of the paired joints is rotated while the other joint is kept still. This strategy is used to reduce redundancy [29]. The DI was defined as the duration ratio of the decomposition movement $[25,26]$.

The objective of this study was to investigate the inter-joint coordination (via the CRP and DI) of the amputated and non-amputated limbs of transfemoral amputees during level walking and to compare it with that for able-bodied individuals. It was hypothesized that the amputated limbs would exhibit a unique CRP pattern and DI as an adaptation to limb salvage, while the non-amputated limbs (owing to the compensatory mechanism to cope with the insufficient biomechanical functions of the amputated limbs) would perform another alteration in the inter-joint coordination. The results of this study facilitate the understanding of motor control of transfemoral amputees and provide important information regarding prosthetic designs and rehabilitation.

\section{Materials and Methods}

\subsection{Participant Information}

Ten unilateral transfemoral amputees were recruited, but two were dismissed owing to a highly unstable walking gait. Eight unilateral transfemoral amputees (age: $30.4 \pm 7.4$ years; height: $1.75 \pm 0.07 \mathrm{~m}$; body mass: $70.3 \pm 9.1 \mathrm{~kg}$ ) were included in the data analysis (Table 1 ). Additionally, eight healthy able-bodied young male adults (age: $26.5 \pm 4.8$ years; height: $1.75 \pm$ 
$0.05 \mathrm{~m}$; body mass: $65.5 \pm 8.3 \mathrm{~kg}$ ) were recruited from the university and participated as controls. The amputees were equipped with prostheses for more than six months. They reported no other gait-affecting pathologies, such as neuromuscular disease. The able-bodied controls reported no history of injuries or disorders that affected their walking gait. The research was fully informed to the participants and signed written consent was obtained. The study was approved by the Human Subject Ethics Sub-committee of The Hong Kong Polytechnic University (Reference No.: HSEARS20170117001).

Table 1. Participant information for the amputee group.

\begin{tabular}{cccccccc}
\hline Code & $\begin{array}{c}\text { Age } \\
\text { (years) }\end{array}$ & $\begin{array}{c}\text { BW } \\
\mathbf{( k g})\end{array}$ & $\begin{array}{c}\text { Height } \\
\mathbf{( m )}\end{array}$ & $\begin{array}{c}\text { Yr. } \\
\text { AMP }\end{array}$ & $\begin{array}{c}\text { Yr. } \\
\text { C.P. }\end{array}$ & Prosthetic Knee & Prosthetic Foot \\
\hline 1 & 23 & 60.3 & 1.68 & 13 & 4 & Jingbo JB-850 & Jingbo FY-YDJ \\
2 & 30 & 54.4 & 1.74 & 1.5 & 1.5 & Ottobock 3R80 & Ottobock 1A30 \\
3 & 24 & 69.5 & 1.88 & 18 & 10 & Jingbo JB-850 & Jingbo FY-YDJ \\
4 & 27 & 66.3 & 1.69 & 5 & 0.5 & Ottobock 3R80 & Ottobock 1S101 \\
5 & 48 & 82.0 & 1.85 & 17 & 6 & Ottobock 3R80 & Ottobock 1C40 \\
6 & 32 & 80.0 & 1.72 & 7 & 5 & Jingbo JB-951 & Jingbo FY-YDJ \\
7 & 32 & 71.8 & 1.7 & 15 & 1 & Jingbo JB-810 & Jingbo FY-YDJ \\
8 & 27 & 78.1 & 1.75 & 5 & 5 & Nabtesco NK-6 & Jingbo FPJ-4 \\
\hline
\end{tabular}

BW: body weight; Yr. AMP: years since amputation; Yr. C.P.: years using their current prosthesis.

\subsection{Experimental Procedure}

Before the experiment, retroreflective markers (diameter of $15 \mathrm{~mm}$ ) were affixed to the surface of the body (Figure 1). According to the Plug-in-Gait Lower Body Marker-set [30], the markers were attached to skeletal landmarks of the posterior/anterior and left/right superior iliac spines, the lower/upper lateral $1 / 3$ surface of the thighs, the lateral femoral epicondyles, the lower/upper lateral $1 / 3$ surface of the shanks, the lateral malleolus, the second metatarsal heads, and the calcaneus for both lower limbs. For amputees, the corresponding markers were attached to the lateral surface with reference to an imaginary line that passed through the rotation axes of the prosthetic knee and ankle.

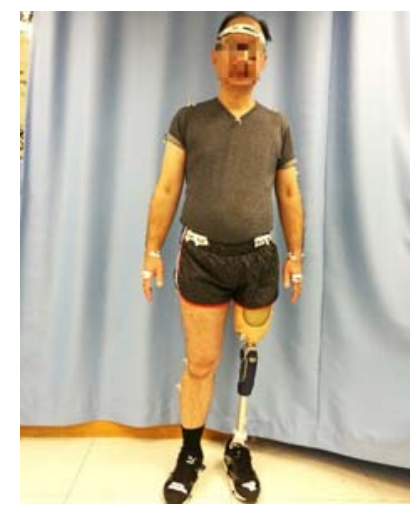

(a)

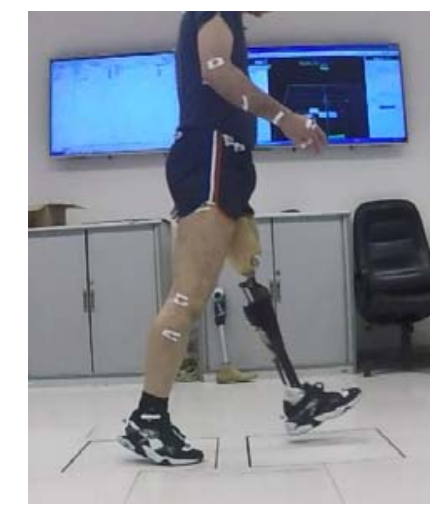

(b)

Figure 1. Illustration of the gait experiment: (a) one of the transfemoral amputee participants, (b) the amputee affixed with markers walking under the motion capture system (MOCAP) and on the force platform.

The participants (both the amputees and the able-bodied control group) were instructed to walk along a $10 \mathrm{~m}$ straight path at a self-selected comfortable speed. All the participants wore tight clothing and sports shoes. An eight-camera MOCAP system (Vicon, Oxford Metrics Ltd., Oxford, United Kingdom) was used to obtain the kinematic data of the markers at a sampling frequency of $200 \mathrm{~Hz}$. Camera calibration was conducted with a residual error of $<0.3 \mathrm{~mm}$. Three successive trials 
were performed for each participant. A successful trial was regarded as a complete capture of the gait cycles for both limbs in a continuous sequence. One set of trial data was randomly selected for subsequent analysis. A fourth-order $6 \mathrm{~Hz}$ low-pass Butterworth filter [21,31] was applied to the data using the software bundled with the MOCAP system (Nexus, Vicon, Oxford Metrics Ltd., Oxford, United Kingdom). Based on the position of the markers, pipelines were built, and a skeleton was reconstructed using the Plug-in-Gait Lower Body model, which was available in the Vicon MOCAP system.

\subsection{Outcome Measures}

The walking speed, stance, and swing time were determined for each group (amputated limb, non-amputated limb, and able-bodied control). The outcome parameters for the able-bodied control group were averaged for the two limbs [21]. The results for the angles and angular velocities of the hip, knee, and ankle joints in the sagittal plane were in time series that were normalized to $100 \%$ of a gait cycle (i.e., 101 data points). We then obtained the phase angle of a joint from the phase portrait according to the normalized joint angle and angular velocity using Equations (1) and (2), as illustrated in Figure 2. The CRP was calculated by subtracting the phase angles of the distal joint from that of the proximal joint $[24,32]$ for three joint pairs: hip-knee, knee-ankle, and hip-ankle, at each time point. The DI of a joint pair was the percentage of time spent in the stance and swing phases when one of the joints was moving while the other was not [25]. The joint was considered "not moving" if the angular velocity was $<10^{\circ} / \mathrm{s}[25,26]$.

$$
\begin{gathered}
g\left(y\left(t_{i}\right)\right)=2\left(\frac{y\left(t_{i}\right)-\min (y(t))}{\max (y(t))-\min (y(t))}\right)-1 \\
f\left(y^{\prime}\left(t_{i}\right)\right)=\frac{y^{\prime}\left(t_{i}\right)}{\max \left(\left|y^{\prime}\left(t_{i}\right)\right|\right)}
\end{gathered}
$$

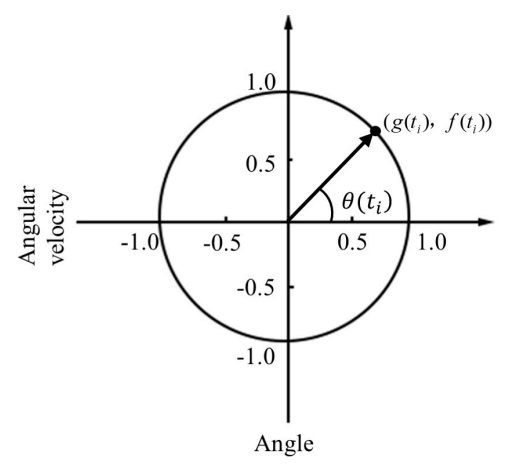

Figure 2. Definition of the phase angle in a phase portrait.

Here, $t_{\mathrm{i}}$ represents time, $y(t)$ represents the joint angle, and $y^{\prime}(t)$ represents the angular velocity. $g(t)$ and $f(t)$ represent the normalized angle and angular velocity, respectively.

\subsection{Data Analysis}

The similarity of the CRP patterns was evaluated according to the cross-correlation coefficients and root-mean-square differences $[23,27,28]$. The mean CRP-time series of each group was calculated. The cross-correlation measures were compared using the ensemble mean curves of the CRP for each group. A large correlation coefficient with a small root-mean-square (RMS) differences indicated good similarity. In this study, the correlation between two CRP curves was considered to be strong when the coefficient was $>0.7$, moderate when the coefficient was $0.3-0.7$, and weak when the coefficient was $<0.3$. The variability of inter-joint coordination within each group was assessed using the deviation 
phase (DP). The standard deviation of the CRP-time series at each time point over the gait cycle was obtained. Subsequently, the DP of a group was calculated as the average of these standard deviation data for all eight CRPs $[17,28]$.

The data process was conducted using a customized MATLAB program (MathWorks Inc., Ismaning, Germany). The normality of the results was checked using the Shapiro-Wilk test (Table 2). If data normality was satisfied, independent sample $t$-tests were used to compare the differences in the walking speeds, stance/swing duration ratios, angular velocities, RMS, DPs, and DIs between the amputated limb and able-bodied control group, and between the non-amputated limb and able-bodied control group. Otherwise, the non-parametric test, Mann-Whitney U test, would be applied. Regarding the analysis of the angular velocities, we performed the $t$-tests on the average angular velocities over midstance, terminal stance, and terminal swing since these comparison outcomes had better implications to DPs and DIs. The level of significance was set as 0.05 . The statistical analysis was performed using SPSS (SPSS Inc., Chicago, IL, USA).

Table 2. Significance level of the Shapiro-Wilk test to check for data normality.

\begin{tabular}{|c|c|c|c|c|c|}
\hline Parameters & Joint Pairs & Phase & $\begin{array}{l}\text { Able-Bodied } \\
\text { Controls }\end{array}$ & $\begin{array}{l}\text { Amputated } \\
\text { Limbs }\end{array}$ & $\begin{array}{c}\text { Non-Amputated } \\
\text { Limbs }\end{array}$ \\
\hline \multirow{6}{*}{ RMS } & \multirow{2}{*}{ Hip-knee } & Stance & 0.24 & 0.65 & 0.78 \\
\hline & & Swing & 0.003 & 0.86 & 0.53 \\
\hline & \multirow{2}{*}{ Knee-ankle } & Stance & 0.16 & 0.09 & 0.55 \\
\hline & & Swing & 0.08 & 0.78 & 0.63 \\
\hline & \multirow{2}{*}{ Hip-ankle } & Stance & 0.08 & 0.81 & 0.78 \\
\hline & & Swing & 0.02 & 0.39 & 0.28 \\
\hline \multirow{6}{*}{$\mathrm{DP}$} & \multirow{2}{*}{ Hip-knee } & Stance & 0.48 & 0.40 & 0.75 \\
\hline & & Swing & $<0.001$ & 0.86 & 0.57 \\
\hline & \multirow{2}{*}{ Knee-ankle } & Stance & 0.22 & 0.07 & 0.49 \\
\hline & & Swing & 0.02 & 0.80 & 0.76 \\
\hline & \multirow{2}{*}{ Hip-ankle } & Stance & 0.10 & 0.81 & 0.86 \\
\hline & & Swing & 0.02 & 0.36 & 0.31 \\
\hline \multirow{6}{*}{ DI } & \multirow{2}{*}{ Hip-knee } & Stance & 0.32 & 0.23 & 0.27 \\
\hline & & Swing & 0.67 & 0.20 & 0.28 \\
\hline & \multirow{2}{*}{ Knee-ankle } & Stance & 0.87 & 0.15 & 0.92 \\
\hline & & Swing & 0.79 & 0.87 & 0.44 \\
\hline & \multirow{2}{*}{ Hip-ankle } & Stance & 0.11 & $<0.001$ & 0.28 \\
\hline & & Swing & 0.71 & 0.57 & 0.19 \\
\hline
\end{tabular}

RMS: root-mean-square; DP: deviation phase; DI: decomposition index. Bold values indicate $p<0.05$.

\section{Results}

\subsection{Walking Speed and Stance-Swing Phase Duration Ratio}

The preferred walking speed of the amputees $(0.92 \pm 0.08 \mathrm{~m} / \mathrm{s})$ was significantly lower than that of the controls $(1.37 \pm 0.13 \mathrm{~m} / \mathrm{s}, p<0.001)$. The duration of the stance phase for the control group accounted for $60.5 \% \pm 1.7 \%$ of the gait cycle, as shown in Figure 3 . The ratio for the non-amputated limb group was significantly larger $(p<0.001): 66.1 \% \pm 2.4 \%$. The amputated limb group exhibited a significantly smaller stance ratio: $58.6 \% \pm 1.2 \%(p<0.001)$. 


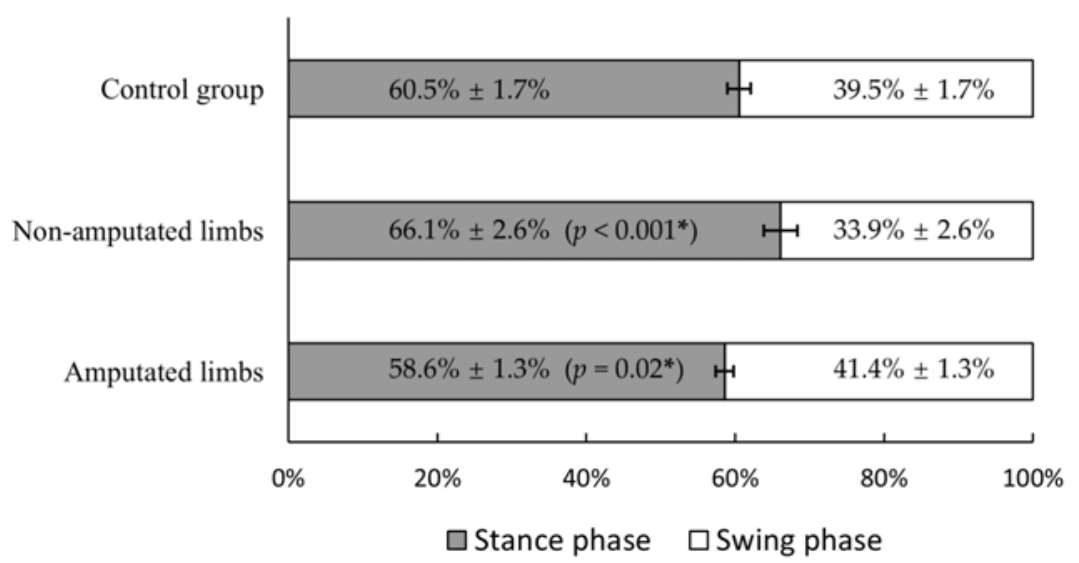

Figure 3. Duration ratio of the stance and swing phases for the amputees with amputated and non-amputated limbs and the able-bodied control group. Significance levels refer to t-test compared to the control group. ${ }^{*}$ indicates a significant difference relative to the control group $(p<0.05)$.

As shown in Figure 4, for the non-amputated limb group, the hip joint was nearly motionless during the terminal swing, with an average angular velocity of $0.6 \pm 1.6^{\circ} / \mathrm{s}$ (flexion) from approximately $91 \%$ to $100 \%$ of the gait cycle. This was significantly lower than the extension velocity for the controls $\left(37.0 \pm 6.2^{\circ} / \mathrm{s}, p<0.001\right)$. An extreme point was observed in the hip angular velocity curve for the amputated limb group. The hip flexion velocity increased after the decline at the frame of $80.8 \% \pm 2.4 \%$ of the gait cycle, which was not observed for the non-amputated limb and control groups. During the mid-stance and terminal stance ( $22 \%$ to $36 \%$ of the gait cycle), the average dorsiflexion velocities of the ankle for the non-amputated limb group were $1.9 \pm 24.1 \%$ s-significantly lower than those for the able-bodied controls $\left(34.8 \pm 30.7^{\circ} / \mathrm{s}, p=0.04\right)$. The source data of joint angles and joint angular velocities were provided in Supplementary Materials of Table S4 and Table S5, respectively.

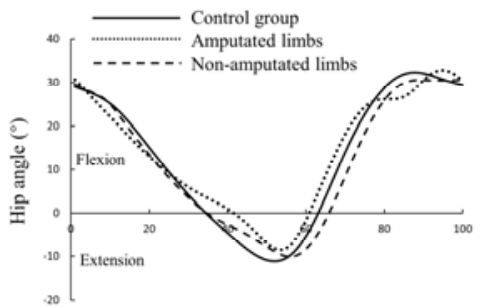

(a)

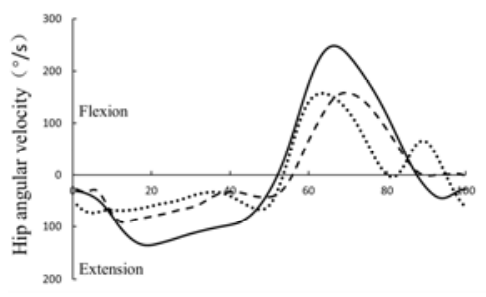

(d)

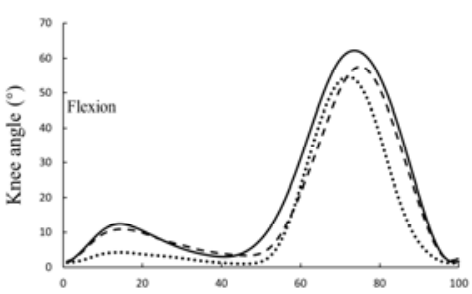

(b)

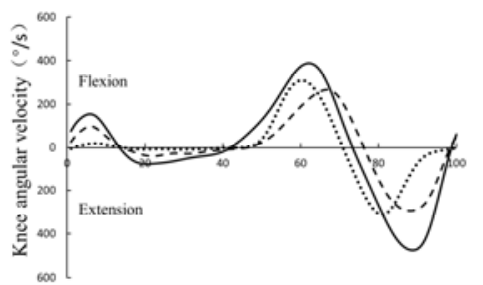

(e)

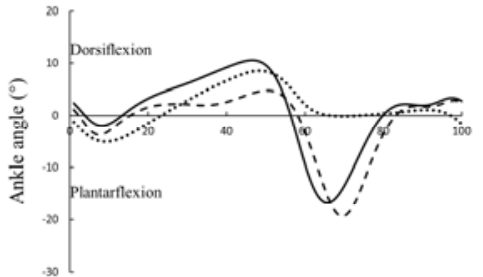

(c)

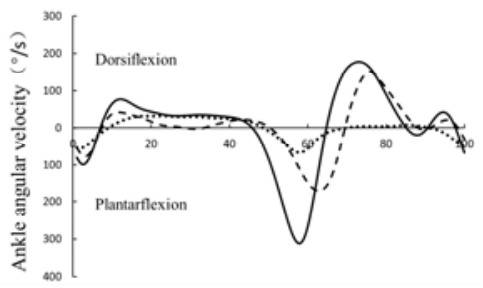

(f)

Figure 4. Comparison of the ensemble mean curves of the angular velocities in the gait cycle: (a) hip joint angle, (b) knee joint angle, (c) ankle joint angle, (d) hip joint angular velocity, (e) knee joint angular velocity, (f) ankle joint angular velocity.

\section{2. $C R P$}

The cross-correlation indicated that the CRP of the non-amputated limb group was significantly correlated to that of the control group ( $p<0.001$ for all the joint pairs), as shown in Table 3 . 
Additionally, the CRP of the non-amputated limb group was strongly correlated to that of the control group (all coefficients $>0.7$ ). Nevertheless, the knee-ankle RMS of the non-amputated limb group was significantly larger than that of the controls in the swing phase: the difference was $18.6 \%(p=0.01)$. No significant difference was observed in the RMS for other joint pairs between the non-amputated limb group and the control group.

Table 3. Cross-correlation coefficients and root-mean-square (RMS) differences between the amputees and able-bodied controls.

\begin{tabular}{cccccc}
\hline \multirow{2}{*}{ Joint Pairs } & Phase & $\begin{array}{c}\text { Amputated } \\
\text { Limb versus } \\
\text { Control }\end{array}$ & $\begin{array}{c}\text { Non-Amputated } \\
\text { Limb versus } \\
\text { Control }\end{array}$ & $\begin{array}{c}\text { Amputated } \\
\text { Limb versus } \\
\text { Control }\end{array}$ & $\begin{array}{c}\text { Non-Amputated } \\
\text { Limb versus } \\
\text { Control }\end{array}$ \\
\hline \multirow{2}{*}{ Hip-knee } & Stance & $0.79(<0.001)$ & $0.89(<0.001)$ & $-3.5 \%(0.41)$ & $3.2 \%(0.93)$ \\
& Swing & $0.79(<0.001)$ & $0.93(<0.001)$ & $-5.4 \%(0.20) \mathrm{M}$ & $-1.7 \%(0.90)^{\mathrm{M}}$ \\
\hline \multirow{2}{*}{ Knee-ankle } & Stance & $-0.05(0.61)$ & $0.78(<0.001)$ & $21.2 \%(0.03)$ & $9.3 \%(0.54)$ \\
& Swing & $0.23(0.02)$ & $0.89(<0.001)$ & $5.0 \%(0.70)$ & $18.6 \%(0.01)$ \\
\hline \multirow{2}{*}{ Hip-ankle } & Stance & $0.296(0.003)$ & $0.81(<0.001)$ & $20.7 \%(0.04)$ & $13.5 \%(0.15)$ \\
& Swing & $0.66(<0.001)$ & $0.88(<0.001)$ & $21.1 \%(0.20) \mathrm{M}$ & $13.8 \%(0.20)$ \\
\hline
\end{tabular}

Brackets refer to significance level; ${ }^{\mathrm{M}}$ Significance level based on the Mann-Whitney $\mathrm{U}$ test because of the data non-normality.

The hip-knee CRP for the amputated limb group was significantly and strongly correlated with that of the controls in stance $(p<0.001$, coefficient $=0.79)$ and swing $(p<0.001$, coefficient $=0.79)$. There were no significant differences in the hip-knee RMS compared to the controls in stance $(p=0.41)$ and swing $(p=0.20)$. The knee-ankle CRP of the amputated limb group was not significantly correlated to that of the controls in the stance phase $(p=0.90)$, and the RMS difference was significant $(21.2 \%$, $p=0.03$ ). Although the knee-ankle CRP of the amputated limb group was significantly correlated to that of the controls during swing $(p=0.02)$ and the RMS relative to the controls was not large $(5 \%$, $p=0.70$ ), the correlation was weak (coefficient $=0.23$ ). The hip-ankle CRP of the non-amputated limb group was significantly correlated to that of the controls in the stance phase $(p=0.003)$, whereas there was a weak correlation (coefficient $=0.296$ ) and the RMS difference was significant $(20.7 \%, p=0.04)$. The hip-ankle CRP of the non-amputated limb group was significantly correlated to that of the controls in the swing phase $(p<0.001)$, with a moderate correlation (coefficient $=0.66$ ). There was no significant difference in the hip-ankle RMS between the amputated limb group and the controls, although the RMS difference was $21.1 \%(p=0.20)$. The source data of RMS among able-bodied and amputees were presented in Supplementary Material Table S1.

The knee-ankle DP of the non-amputated limb group was significantly higher than that of the controls during swing $(66.8 \pm 5.6$ versus $54.9 \pm 10.7, p=0.01)$, as shown in Figure 5 . No significant difference in the DP of other joint pairs was observed between the non-amputated limb group and the controls.

There was no significant difference in the hip-knee DP between the amputated limb group and the controls in the stance phase. However, the knee-ankle DP and hip-ankle DP of the amputated limb group were significantly different from those of the controls in the stance phase. The knee-ankle DP of the amputated limb group was 9.1 higher than that of the controls $(40.7 \pm 6.3$ versus $31.6 \pm 4.7$, $p=0.01$ ), and the hip-ankle DP was 6.2 higher ( $56.2 \pm 7.0$ versus $46.4 \pm 10.2, p=0.04)$. No significant difference was observed in the DPs of other joint pairs during the swing phase between the amputated limb group and the controls. The source data of DP among able-bodied and amputees were presented in Supplementary Material Table S2. 


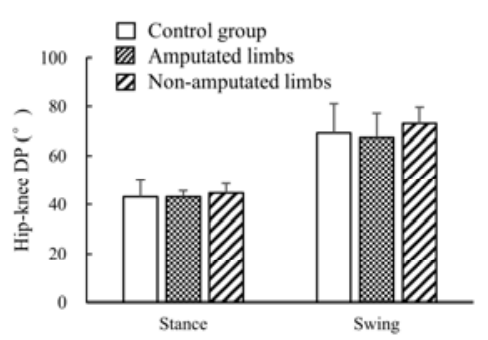

(a)

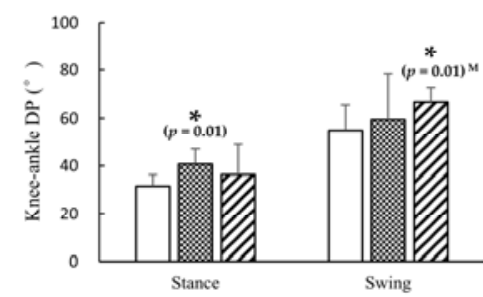

(b)

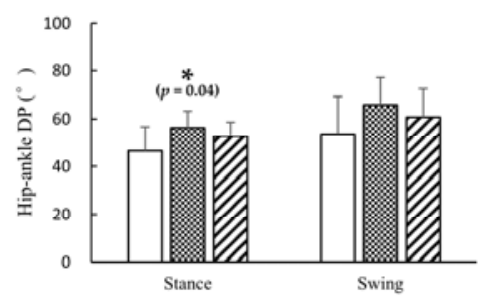

(c)

Figure 5. Comparison of the deviation phase (DP): (a) hip-knee, (b) knee-ankle, (c) hip-ankle. * indicates a significant difference relative to the control group $(p<0.05)$.

\section{3. $D I$}

Overall, the amputees exhibited a higher DI than the controls, as shown in Figure 6. There was no significant difference in the hip-knee DI between the non-amputated limb group and the controls in stance, whereas the DI was significantly larger in the swing phase $(25.5 \pm 16.3$ versus $8.3 \pm 4.7$, $p=0.02$ ). No significant difference was observed in the knee-ankle DI and hip-ankle DI between the non-amputated limb group and the controls in stance and swing.

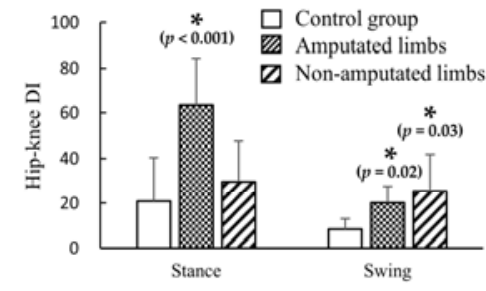

(a)

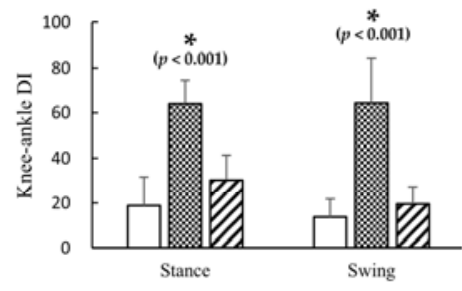

(b)

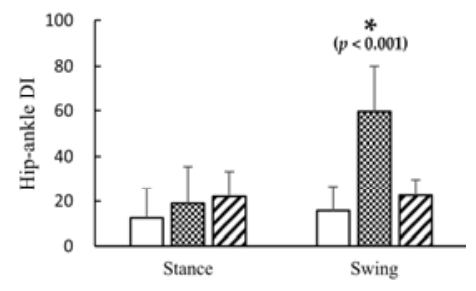

(c)

Figure 6. Comparison of the decomposition index (DI): (a) hip-knee, (b) knee-ankle, (c) hip-ankle.

* indicates a significant difference compared to the control group.

The hip-knee DI of the amputated limb group was significantly larger than that of the controls in the stance $(63.3 \pm 20.9$ versus $20.9 \pm 19.5, p<0.001)$ and swing phases $(20.1 \pm 7.5$ versus $8.3 \pm 4.7$, $p=0.002)$. The knee-ankle DI of the amputated limb group was significantly larger than that of the controls in stance $(63.9 \pm 10.7$ versus $18.7 \pm 12.9, p<0.001)$ and swing $(64.3 \pm 20.1$ versus $13.7 \pm 8.1$, $p<0.001)$. The hip-ankle DI of the amputated limb group was not significantly different from that of the controls in the stance phase, but it was significantly larger in the swing phase ( $59.7 \pm 20.3$ versus $15.6 \pm 10.9, p<0.001)$. The source data of DI among able-bodied and amputees were presented in Supplementary Material Table S3.

\section{Discussion}

In this study, we investigated the inter-joint coordination of the amputated and non-amputated limbs for eight unilateral transfemoral amputees and compared them with the inter-joint coordination for eight able-bodied participants. The added value of this study was to target the inter-joint coordination, whereas previous studies often focused on the kinematics or muscle electromyography (EMG) of the transfemoral gait. The inter-joint coordination during level walking was quantified by CRP and DI. Our results indicated that there were differences in the inter-joint coordination for both the amputated and non-amputated limb groups compared with the able-bodied controls. This was mainly observed by changing the relative phase angles and adapting to a higher degree of decomposition movement between the hip-knee, knee-ankle, and hip-ankle joint couples. The results supported our hypothesis that the prosthetic mechanism contributed to the accommodated inter-joint coordination, and the non-amputated limbs adjust the control strategy to fulfill the requirements of a prosthetic gait 
via a compensatory mechanism. This finding is significant because it elucidates the motor control strategy for transfemoral amputees and provides a more in-depth view of their insufficient athletic ability. Thus, this study can facilitate improvements in rehabilitation and prosthetic designs.

The inter-joint coordination between the amputated limbs and the controls was significantly different (with regard to the CRP) for the knee-ankle and hip-ankle joints. The CRPs were only weakly or moderately correlated. The findings reflected the attenuation of the control strategy upon limb salvage. Because the prosthetic knee joint did not resist flexion in the normal range, either the amputees or the prosthetist adjusted the knee joints to a higher level of extension or hyper-extension to withstand the body support during the single support phase. Moreover, the kinematics of the prosthetic ankle joint were different from those of the human ankle [33]. These factors contributed to the differences in the joint angles and angular velocities and thus the differences in coordination between the amputated limb group and the able-bodied control group.

Despite the fact that the non-amputated limb group exhibited an intact system without limb salvage, the knee-ankle CRP of the non-amputated limb group was significantly different from that of the able-bodied control group during swing, with an RMS difference of $18.6 \%(p=0.01)$. The swing phase of the non-amputated limbs indicated that the amputated limbs were in a single support phase. Schaarschmidt et al. [2] investigated the gait asymmetry of unilateral transfemoral amputees and reported that the amputated limbs had a poor body support ability in the single support phase. Based on our findings, we anticipated that the amputees adjusted the movement of the distal segments (foot and shank) of the non-amputated limb as a form of compensation to maintain a dynamic balance during walking.

Our findings revealed significant differences in the DI for the majority of the comparisons, particularly for the proximal joints (hip joint). The hip joint of the non-amputated limb group nearly came to a stop during the last $10 \%$ of the gait cycle, which contributed to the large DI. This phenomenon may facilitate early preparation for the next gait cycle to accommodate the weakened single support of the amputated limbs, which was evidenced by the shortened swing phase in our study. The hip joint of the amputated limb was the only limb part without salvage and produced an active power to drive the residual limb and the suspended prosthesis. The hip joint coordinated other distal joints to ensure accurate footstep positions, particularly during the terminal swing. Because of the essential roles, the hip joints of both limbs decomposed the movement and coordinated with the knee joints, which significantly increased the DI, which was in agreement with existing studies that the amputees predominantly regulated the hip joints for balance and walking stability $[16,34,35]$.

The amputees needed to utilize and coordinate the ankle joint of the non-amputated side at a comparatively lower flexibility than the able-bodied controls, particularly during the midstance and terminal stance. Bastian et al. [25] studied patients with cerebellar ataxia and concluded that reducing the speed of movement and adopting decomposition moments can increase the accuracy of movement, which is a manifestation of compensatory gait. Increasing the knee-ankle DI of the non-amputated limb can yield a stable and accurate gait at the cost of the walking speed.

Different prosthetic designs can have different effects on the inter-joint coordination of the amputated limb, resulting in a higher DP for the amputee groups. In this study, the prostheses were not identical. Amputees wore their own daily used prostheses, walking at their preferred comfortable speeds. The knee-ankle DP and hip-ankle DP of the amputated limbs were $9.1^{\circ}$ and $6.2^{\circ}$ higher than those of the controls in the stance phase, respectively. Different prosthetic joints can have different characteristics and mechanisms. Solid ankle cushion heel (SACH), multi-axis ankle-foot, and energy-storing-and-returning (ESR) feet were used in this study. The SACH foot consisted of a rigid ankle joint, while the multi-axis ankle-foot allowed rotation in multiple directions. The ESR foot did not contain a real ankle joint, but the functions were partially replicated by the deformation of the material. The different mechanisms may lead to variations in the joint angle [33] and angular velocity. This may have caused differences in the knee-ankle and hip-ankle CRPs among the amputees. 
Thus, the amputee groups exhibited higher DPs (which indicated the variation among subjects) than the control group.

This study had limitations. First, all the participants were male. Second, inter-joint coordination was investigated only in the sagittal plane. Information on the coronal and transverse planes could be essential to establish a more complete inter-joint coordination profile for amputees. Moreover, the walking speed was not controlled, because amputees may have adopted their natural gait at different walking speeds. The amputees walked slower than the able-bodied controls in our experiment, which may have contributed to covariances. In addition to the inter-joint coordination, the interaction between the asymmetry and the variability can be investigated as a manifestation of gait instability [36]. Additionally, it would be interesting to know whether wearables with feedback installed on the prosthesis can improve the gait symmetry and inter-joint coordination [37-39].

\section{Conclusions}

Unilateral transfemoral amputees utilized different inter-joint coordination patterns in the amputated and non-amputated limbs to ensure foot placement accuracy and compensate for the impaired support of the amputated side respectively, which was primarily regulated by adapting a higher degree of decomposition movement at the hip joints. In future studies, researchers should investigate whether muscle strengthening for enhancing the support capability and motor control training for foot placement accuracy can improve the inter-joint coordination and symmetry. Besides, the use of portable or wearable sensors, such as an inertial measurement unit (IMU), could facilitate the measurement of three-dimensional kinematics and gait asymmetry in clinical settings [39].

Supplementary Materials: Source data including those underlying Figure 4, Figure 5, and Figure 6, are available in the supplementary materials. The following are available online at http://www.mdpi.com/2076-3417/10/12/4072/s1, Table S1. RMS of the able-bodied controls, amputated limbs and non-amputated limbs of amputees; Table S2. DP of the able-bodied controls, amputated limbs and non-amputated limbs of amputees; Table S3. DI of the able-bodied controls, amputated limbs and non-amputated limbs of amputees; Table S4. Source data of joint angles among able-bodied and amputees; Table S5. Source data of joint angular velocities among able-bodied and amputees.

Author Contributions: Conceptualization, Z.X.; methodology, Z.X., D.W.-C.W., and F.Y.; software, Z.X.; validation, D.W.-C.W.; formal analysis, F.Y.; investigation, Z.X. and D.W.-C.W.; resources: M.Z. and Y.-B.F.; data curation: Z.X. and T.L.-W.C.; writing - original draft preparation, Z.X.; writing - review and editing, Z.X., D.W.-C.W., and F.Y.; visualization, Z.X.; supervision, W.-T.J.; project administration, Z.X. and D.W.-C.W.; funding acquisition, M.Z. and W.-T.J. All authors have read and agreed to the published version of the manuscript.

Funding: This research was funded by the Key Program of the Ministry of Science and Technology of China (2017YFB0702500), the National Natural Science Foundation of China (11972239, 11772210), and a research studentship from The Hong Kong Polytechnic University and Sichuan University.

Conflicts of Interest: None of the authors will benefit from any commercial group with this work. No financial support was received or will be received by any of the authors for publication of this article.

\section{References}

1. Esposito, E.R.; Whitehead, J.M.A.; Wilken, J. Sound limb loading in individuals with unilateral transfemoral amputation across a range of walking velocities. Clin. Biomech. 2015, 30, 1049-1055. [CrossRef] [PubMed]

2. Schaarschmidt, M.; Lipfert, S.; Meier-Gratz, C.; Scholle, H.-C.; Seyfarth, A. Functional gait asymmetry of unilateral transfemoral amputees. Hum. Mov. Sci. 2012, 31, 907-917. [CrossRef] [PubMed]

3. Esposito, E.R.; Rábago, C.A.; Wilken, J. The influence of traumatic transfemoral amputation on metabolic cost across walking speeds. Prosthetics Orthot. Int. 2017, 42, 214-222. [CrossRef] [PubMed]

4. Levine, D.; Richards, J.; Whittle, M. Whittle's Gait Analysis; Elsevier LTD: Oxford, UK, 2012.

5. Drevelle, X.; Villa, C.; Bonnet, X.; Loiret, I.; Fodé, P.; Pillet, H. Vaulting quantification during level walking of transfemoral amputees. Clin. Biomech. 2014, 29, 679-683. [CrossRef] 
6. Segal, A.D.; Orendurff, M.S.; Klute, G.; McDowell, M.L.; Pecoraro, J.A.; Shofer, J.; Czerniecki, J.M. Kinematic and kinetic comparisons of transfemoral amputee gait using C-Leg and Mauch SNS prosthetic knees. J. Rehabilitation Res. Dev. 2007, 43, 857. [CrossRef] [PubMed]

7. Hobara, H.; Kobayashi, Y.; Nakamura, T.; Yamasaki, N.; Nakazawa, K.; Akai, M.; Ogata, T. Lower extremity joint kinematics of stair ascent in transfemoral amputees. Prosthetics Orthot. Int. 2011, 35, 467-472. [CrossRef]

8. Schmalz, T.; Blumentritt, S.; Marx, B. Biomechanical analysis of stair ambulation in lower limb amputees. Gait Posture 2007, 25, 267-278. [CrossRef]

9. Morgenroth, D.; Roland, M.; Pruziner, A.L.; Czerniecki, J.M. Transfemoral amputee intact limb loading and compensatory gait mechanics during down slope ambulation and the effect of prosthetic knee mechanisms. Clin. Biomech. 2018, 55, 65-72. [CrossRef]

10. Carse, B.; Scott, H.; Brady, L.; Colvin, J. A characterisation of established unilateral transfemoral amputee gait using 3D kinematics, kinetics and oxygen consumption measures. Gait Posture 2019, 75, 98-104. [CrossRef]

11. Vitali, A.; Colombo, G.; Regazzoni, D.; Rizzi, C. Virtual prototyping and physical experimentation of lower limb prosthesis. In Proceedings of the 5th Workshop on ICTs for improving Patients Rehabilitation Research Techniques, Popayán, Colombia, 11-13 September 2019; pp. 111-117.

12. Pauw, K.D.; Serrien, B.; Baeyens, J.-P.; Cherelle, P.; Bock, S.D.; Ghillebert, J.; Bailey, S.P.; Lefeber, D.; Roelands, B.; Vanderborght, B.; et al. Prosthetic gait of unilateral lower-limb amputees with current and novel prostheses: A pilot study. Clin. Biomech. 2020, 71, 59-67. [CrossRef]

13. Wong, D.W.-C.; Lam, W.-K.; Yeung, L.; Lee, W.C.; Lee, C.C.W. Does long-distance walking improve or deteriorate walking stability of transtibial amputees? Clin. Biomech. 2015, 30, 867-873. [CrossRef] [PubMed]

14. St-Onge, N.; Feldman, A.G. Interjoint coordination in lower limbs during different movements in humans. Exp. Brain Res. 2003, 148, 139-149. [CrossRef] [PubMed]

15. Iosa, M.; Marro, T.; Paolucci, S.; Morelli, D. Stability and harmony of gait in children with cerebral palsy. Res. Dev. Disabil. 2012, 33, 129-135. [CrossRef] [PubMed]

16. A Winter, D. Foot Trajectory in Human Gait: A Precise and Multifactorial Motor Control Task. Phys. Ther. 1992, 72, 45-53. [CrossRef]

17. Lu, T.-W.; Yen, H.-C.; Chen, H.-L. Comparisons of the inter-joint coordination between leading and trailing limbs when crossing obstacles of different heights. Gait Posture 2008, 27, 309-315. [CrossRef] [PubMed]

18. Yen, H.-C.; Chen, H.-L.; Liu, M.-W.; Liu, H.-C.; Lu, T.-W. Age effects on the inter-joint coordination during obstacle-crossing. J. Biomech. 2009, 42, 2501-2506. [CrossRef] [PubMed]

19. Chiu, S.-L.; Chou, L.-S. Effect of walking speed on inter-joint coordination differs between young and elderly adults. J. Biomech. 2012, 45, 275-280. [CrossRef]

20. Cowley, J.; Gates, D.H. Inter-joint coordination changes during and after muscle fatigue. Hum. Mov. Sci. 2017, 56, 109. [CrossRef]

21. Chiu, S.-L.; Chang, C.-C.; Chou, L.-S. Inter-joint coordination of overground versus treadmill walking in young adults. Gait Posture 2015, 41, 316-318. [CrossRef]

22. Doherty, C.; Bleakley, C.; Hertel, J.; Caulfield, B.; Ryan, J.; Sweeney, K.; Delahunt, E. Inter-joint coordination strategies during unilateral stance following first-time, acute lateral ankle sprain: A brief report. Clin. Biomech. 2015, 30, 636-639. [CrossRef]

23. Wang, T.-M.; Yen, H.-C.; Lu, T.-W.; Chen, H.-L.; Chang, C.-F.; Liu, Y.-H.; Tsai, W.-C. Bilateral knee osteoarthritis does not affect inter-joint coordination in older adults with gait deviations during obstacle-crossing. J. Biomech. 2009, 42, 2349-2356. [CrossRef]

24. Lamb, P.; Stöckl, M. On the use of continuous relative phase: Review of current approaches and outline for a new standard. Clin. Biomech. 2014, 29, 484-493. [CrossRef] [PubMed]

25. Bastian, A.J.; Martin, T.A.; Keating, J.G.; Thach, W.T. Cerebellar ataxia: Abnormal control of interaction torques across multiple joints. J. Neurophysiol. 1996, 76, 492-509. [CrossRef] [PubMed]

26. Earhart, G.M.; Bastian, A.J. Selection and coordination of human locomotor forms following cerebellar damage. J. Neurophysiol. 2001, 85, 759-769. [CrossRef]

27. Haddad, J.M.; Van Emmerik, R.E.; Whittlesey, S.N.; Hamill, J. Adaptations in interlimb and intralimb coordination to asymmetrical loading in human walking. Gait Posture 2006, 23, 429-434. [CrossRef] [PubMed]

28. Chiu, S.-L.; Lu, T.-W.; Chou, L.-S. Altered inter-joint coordination during walking in patients with total hip arthroplasty. Gait Posture 2010, 32, 656-660. [CrossRef] 
29. Morton, S.M.; Bastian, A.J. Relative Contributions of Balance and Voluntary Leg-Coordination Deficits to Cerebellar Gait Ataxia. J. Neurophysiol. 2003, 89, 1844-1856. [CrossRef]

30. Kim, J.H.; Lee, K.-K.; Kong, S.J.; An, K.O.; Jeong, J.H.; Lee, Y.S. Effect of Anticipation on Lower Extremity Biomechanics During Side- and Cross-Cutting Maneuvers in Young Soccer Players. Am. J. Sports Med. 2014, 42, 1985-1992. [CrossRef]

31. Hutin, É.; Pradon, D.; Barbier, F.; Bussel, B.; Gracies, J.-M.; Roche, N. Walking velocity and lower limb coordination in hemiparesis. Gait Posture 2012, 36, 205-211. [CrossRef]

32. Burgess-Limerick, R.; Abernethy, B.; Neal, R.J. Relative phase quantifies interjoint coordination. J. Biomech. 1993, 26, 91-94. [CrossRef]

33. Versluys, R.; Beyl, P.; Van Damme, M.; Desomer, A.; Van Ham, R.; Lefeber, D. Prosthetic feet: State-of-the-art review and the importance of mimicking human ankle-foot biomechanics. Disabil. Rehabil. Assist. Technol. 2009, 4, 65-75. [CrossRef] [PubMed]

34. Monno, A.; Temprado, J.; Zanone, P.-G.; Laurent, M. The interplay of attention and bimanual coordination dynamics. Acta Psychol. 2002, 110, 187-211. [CrossRef]

35. Nolan, L.; Wit, A.; Dudziñski, K.; Lees, A.; Lake, M.; Wychowañski, M. Adjustments in gait symmetry with walking speed in trans-femoral and trans-tibial amputees. Gait Posture 2003, 17, 142-151. [CrossRef]

36. Wong, D.W.-C.; Lam, W.-K.; Lee, W.C.-C. Gait asymmetry and variability in older adults during long-distance walking: Implications for gait instability. Clin. Biomech. 2020, 72, 37-43. [CrossRef] [PubMed]

37. Ma, C.Z.-H.; Wan, A.H.-P.; Wong, D.W.-C.; Zheng, Y.; Lee, C.C.W. A Vibrotactile and Plantar Force Measurement-Based Biofeedback System: Paving the Way towards Wearable Balance-Improving Devices. Sensors 2015, 15, 31709-31722. [CrossRef]

38. Wan, A.H.; Wong, D.W.-C.; Ma, C.Z.-H.; Zhang, M.; Lee, C.C.W. Wearable Vibrotactile Biofeedback Device Allowing Identification of Different Floor Conditions for Lower-Limb Amputees. Arch. Phys. Med. Rehabil. 2016, 97, 1210-1213. [CrossRef]

39. Glowinski, S.; Łosiński, K.; Kowiański, P.; Waśkow, M.; Bryndal, A.; Grochulska, A. Inertial Sensors as a Tool for Diagnosing Discopathy Lumbosacral Pathologic Gait: A Preliminary Research. Diagn. 2020, 10, 342. [CrossRef]

(C) 2020 by the authors. Licensee MDPI, Basel, Switzerland. This article is an open access article distributed under the terms and conditions of the Creative Commons Attribution (CC BY) license (http://creativecommons.org/licenses/by/4.0/). 